\title{
Conciliation emploi-famille et porosité des temps sociaux chez les avocats et les avocates : des stratégies de report et d'intensification pour arriver à concilier?
}

\author{
Diane-Gabrielle Tremblay
}

Our research focuses on verifying to what extent the practice of law has evolved over the years with regard to balancing work and family in different work environments and types of practice. We chose to do qualitative research, using interviews, in order to better understand the career paths of individuals and the reasons for their professional and familial choices. We were able to determine that lawyers often benefit from more autonomy and greater flexibility in organizing their work time. However, when they start having children and they have to balance work with family life, lawyers - especially women - develop strategies to "meet billable hour targets" and perform better, without infringing too much on their personal or family time. This can be achieved not only by deferring tasks but also by intensifying time spent at the office, eliminating lunch breaks, using voice mail, eliminating coffee breaks and exchanging with colleagues, and so on. The interference of work on family/personal life is not always experienced negatively by lawyers because it seems to be internalized by everyone. One may certainly wonder about the impact of these strategies for managing work time (intensification, deferring) on careers.

Notre recherche visait à vérifier dans quelle mesure la pratique de la profession d'avocat a évolué au fil des ans pour permettre de concilier emploi et famille et ce, dans les différents milieux de travail et types de pratique. Nous avons choisi de faire une recherche qualitative, par entretiens, afin de mieux comprendre les trajectoires des personnes et les motifs de leurs choix professionnels et familiaux. Nous avons pu constater que les avocats bénéficient souvent d'une grande autonomie et d'une bonne flexibilité dans l'organisation de leur temps de travail. Par contre, lorsqu'arrivent les enfants et qu'il faut concilier le travail avec la vie familiale, les avocats, surtout les femmes, développent des stratégies pour "faire les heures facturables », mieux performer, sans trop déborder sur leur temps personnel ou familial. Cela peut se faire par le report d'activités, mais aussi par l'intensification du travail au bureau, élimination de la pause de dîner, recours au répondeur, suppression des pauses café, des échanges avec les collègues, etc. L'interférence du travail sur la vie familiale/ personnelle n'est pas toujours vécue négativement par les avocats, parce qu'elle 
semble intériorisée par tous. On peut certes s'interroger sur l'effet de ces stratégies d'aménagement du temps de travail (intensification, report) sur les carrières.

\section{Introduction et problématique théorique}

Le conflit et la conciliation emploi-famille sont des thèmes qui ont suscité beaucoup d'intérêt au cours des dernières décennies. Greenhaus et Beutell ${ }^{1}$ définissent le « conflit emploi-famille » comme l'incompatibilité entre les exigences de l'emploi et les obligations familiales, faisant en sorte que l'implication dans un rôle rend difficile l'engagement dans l'autre. Le conflit apparaît lorsque l'individu perçoit les attentes de son rôle familial comme contradictoires avec les attentes de son rôle professionnel, et vice versa. ${ }^{2}$ Les mesures de conciliation visent alors à diminuer ce conflit, à réduire les difficultés d'articulation et à faciliter l'organisation des temps et des responsabilités des salariés.

Selon Simkin et Hillage, ${ }^{3}$ les politiques de conciliation emploi-famille incluent l'ensemble des mesures formelles et informelles visant à aider les employés à combiner leurs responsabilités familiales et professionnelles. Scheibl et Dex ${ }^{4}$ ajoutent la notion de synergie, pour souligner que ces politiques sont avantageuses pour les employées mais aussi pour les employeurs, et la notion d'équité, selon laquelle de telles politiques devraient en effet permettre aux femmes comme aux hommes de mieux concilier emploi et famille, quel que soit leur domaine d'emploi.

Un certain nombre d'études font état du manque de temps exprimé par les parents de jeunes enfants, principalement ceux de moins de six ans, mais aussi d'adolescents. ${ }^{5}$ Par ailleurs, d'autres travaux ont montré que les mesures les plus recherchées pour faciliter la conciliation tournent autour des mesures temporelles ou touchant les horaires de travail, ou encore des mesures spatiales, comme le fait de pouvoir travailler chez soi à l'occasion. ${ }^{6}$

1. Jeffrey H. Greenhaus et Nicholas Beutell, « Sources of Conflict between Work and Family Roles » (1985) 10:1 Academy of Management Review 76.

2. Michale R Frone, et Robert W Rice, « Work-family Conflict: The Effect of Job and Family Involvement » (1986) 8 Journal of Occupational Behaviour 45.

3. Clare Simkin et James Hillage, Family-friendly Working : New Hope or Old Hype, Brighton, Institute for Manpower Studies, 1992.

4. Fiona Scheibl et Shirley Dex, « Should We Have More Family-Friendly Policies? » (1998) 16:5 European Management Journal 586.

5. Conference Board of Canada, Concilier le travail et la famille : enjeux et options, Ottawa, Conference Board of Canada, 1994; Statistique Canada, Au fil des heures . . . L'emploi du temps des Canadiens par Judith Frederick, Ottawa, Statistique Canada, 1995; Ellen Galinsky, Stacy S. Kim, et James T. Bond, Feeling Overworked : When Work Becomes Too Much-Executive Summary, New York, Families and Work Institute, 2001; Gregory K Stephens et Steven M Sommer, « The Measurement of Work to Family Conflict » (1996) 56:3 Educational and Psychological Measurement 475.

6. Diane-Gabrielle Tremblay, « Paid Parental Leave : An Employee Right or Still an Ideal ? The Situation in Québec and in Canada » (2010) 22:2 Employee Responsibilities and Rights Journal 83. 
Guérin et al. ${ }^{7}$ ont observé que la culture organisationnelle influence le degré de conflit emploi-famille alors que Tremblay ${ }^{8}$ a montré qu'un soutien concret (et non seulement de principe) du conjoint et du supérieur/employeur peut contribuer à diminuer les difficultés de conciliation. Ainsi, les difficultés sont réduites lorsque l'employé constate que son supérieur manifeste de l'empathie ou accepte des accommodements afin de faciliter l'articulation de la vie professionnelle avec la vie familiale. Chenevier ${ }^{9}$ recense trois études faisant état de l'influence de l'attitude négative ou du manque de flexibilité du supérieur sur l'employé quant au conflit emploi-famille : de façon générale, une attitude négative de la part du supérieur stresse l'employé, le rend insatisfait et a pour conséquence qu'il s'absente plus souvent de son travail, voire songe à quitter. Selon l'étude de Caussignac, ${ }^{10}$ l'appui organisationnel aide significativement à diminuer le conflit emploi-famille.

Certains travaux montrent que les gestionnaires et les professionnels se sentent beaucoup plus surchargés de travail que les autres employés; le nombre d'heures qu'ils consacrent au travail rémunéré est significativement plus élevé que celui des autres groupes ${ }^{11}$. Duxbury et al. ont aussi noté que les personnes occupant un emploi professionnel travaillent un plus grand nombre d'heures que les autres groupes de travailleurs, ce qui peut amplifier le conflit emploi-famille. ${ }^{12}$ Elliott, Dale et Egerton ${ }^{13}$ ont pour leur part indiqué qu'il était plus facile pour les femmes non qualifiées de concilier l'emploi et la famille, comparativement aux professionnelles, mais il n'y a pas consensus sur cette question, certains travaux indiquant que les femmes moins qualifiées ont moins d'autonomie et moins de possibilités de négocier avec leur supérieur. ${ }^{14}$ Les milieux professionnels sont par contre caractérisés par un fort éthos professionnel, des normes professionnelles souvent fortes, et c'est ce qui nous a amenée à nous intéresser à une profession souvent fort exigeante, celle d'avocat.

7. Gilles Guérin, Sylvie St-Onge, Laure Chevalier, Kathy Denault et Martine Deschamps, Le conflit emploi-famille: ses causes et ses conséquences : résultats d'enquête, Montréal, Université de Montréal, 1997.

8. Diane-Gabrielle Tremblay, « Articulation emploi-famille : les sources de difficultés et les perspectives de solution dans les secteurs de l'éducation, de la santé et des services sociaux » (2005) Revue multidisciplinaire sur l'emploi, le syndicalisme et le travail 36.

9. Laure Chenevier, Les variables influençant l'ampleur du conflit « emploi-famille » ressenti par l'employé(e), mémoire de maitrise, École des Hautes Études commerciales, Montréal, 1996.

10. Émilie Caussignac, La nature des liens entre les déterminants du conflit emploi-famille, son ampleur et ses impacts, mémoire de maitrise, École des Hautes Études commerciales, Montréal, 2000.

11. Galinsky, supra note 5 .

12. Linda Duxbury, Christopher Higgins et C. Lee, « The Impact of Job Type and Family Type on Work-Family Conflict and Perceived Stress: A Comparative Analysis » (1993) 14: 9 Ressources humaines 21 .

13. Jane Elliott, Angela Dale, et Muriel Egerton, « The Influence of Qualifications on Women's Work Histories, Employment Status and Occupational Attainment at Age $33 »$ (2001) 17:2 European Sociological Review 145.

14. Diane-Gabrielle Tremblay, Conciliation emploi-famille et temps sociaux, $3^{\mathrm{e}}$ éd, Québec, Presses de l’Université du Québec, 2012. 
Un bon nombre d'études et de publications aux États-Unis ont d'ailleurs indiqué que plusieurs femmes professionnelles choisissent de quitter leur profession, en raison de difficultés trop grandes et de l'absence de flexibilité des milieux de travail ${ }^{15}$. Par contre, nous pensons que si cette réalité existe aussi au Québec, elle est nettement moins présente que cela ne semble être le cas aux États-Unis ou peut-être même au Canada anglais, en raison de l'importance de la politique familiale au Québec, et surtout de la politique des services de garde à coûts réduits $(7 \$$ par jour) et des congés parentaux bien rémunérés ${ }^{16}$. Si ces politiques ne sont pas parfaites, elles soutiennent tout de même mieux les mères en emploi que ce n'est le cas ailleurs en Amérique, de sorte que les femmes vont peut-être réorienter leur carrière ou changer de milieu de travail, mais pas nécessairement quitter la profession (Opting Out). Certaines études montrent que si des hommes quittent aussi la carrière ascensionnelle (« fast lane »), ils le font pour des raisons différentes des femmes. Ainsi, Hewlett et Luce ${ }^{17}$ ont montré que les femmes étaient $44 \%$ à quitter pour des raisons familiales, alors que lorsque les hommes abandonnent leurs aspirations pour les carrières promotionnelles (devenir associé par exemple dans le cas des avocats), c'est davantage ( $29 \%$ ) pour opter vers un autre type de milieu de travail. Les motifs familiaux jouent aussi dans les réorientations de carrière au Québec, comme nous le verrons.

Quelques recherches menées auprès des avocats font tout de même état d'une difficulté à monter dans les organisations et parfois même à simplement demeurer dans un grand cabinet. Nombre d'études montrent que la profession juridique impose un investissement temporel important ${ }^{18}$ qui peut nuire à la conciliation. Cela

15. Lisa Belkin, " The Opt-Out Revolution », New York Times Magazine (26 octobre 2003) 42 ; Heather Boushey, " "Opting Out?" The Effect of Children on Women's Employment in the United States » (2008) 14:1 Feminist Economics 1; Christine Percheski, « Opting Out? Cohort Differences in Professional Women's Employment Rates from 1960 to 2005 » (2008) 73:3 American Sociological Review 497.

16. Diane-Gabrielle Tremblay et Émilie Genin, « Parental Leave: When First Hand Experience Does Not Measure Up to Perception » (2010) 30:9/10 International Journal of Sociology and Social Policy 532 .

17. Sylvia A Hewlett et Carolyn B Luce, « Off-Ramps and On-Ramps: Keeping Talented Women on the Road to Success » (2005) 83 Harvard Business Review 43.

18. Joan Brockman, Gender in the Legal Profession: Fitting or Breaking the Mould, Vancouver, University of British Columbia Press, 2001; Joan Brockman, « The Use of Self-regulation to Curb Discrimination and Sexual Harassment in the Legal Profession » (1997) 35:2 Osgoode Hall Law Journal 209; Joan Brockman, « Leaving the Practice of Law : The Wherefores and the Whys » (1994) 32:1 Alberta Law Review 116 ; Joan Brockman, « Resistance by the Club to the Feminization of the Legal Profession » (1992) 7:2 Canadian Journal of Law Society 47 ; Fiona Kay, Cristi Masuch, et Paula Curry, Diversity and Change : The Contemporary Legal Profession in Ontario, Toronto, Law Society of Upper Canada, 2004; Fiona Kay, « Crossroads to Innovation and Diversity : The Careers of Women Lawyers in Quebec » (2002) 47 McGill Law Journal 699; Cynthia Epstein, « Border Crossings: The Constraints of Time Norms in Transgressions of Gender and Professional Roles » dans Cynthia Epstein et Arne Kalleberg, dir, Fighting for Time: Shifting Boundaries of Work and Social Life, New York, Russell Sage, 2004, 317; Cynthia Epstein, Women in Law, New York, Basic Books, 1981; Cynthia Epstein et Carol Seron, « The Symbolic Meanings of Professional Time ", dans Jerry Van Hoy, Legal Professions : Work, 
représente donc un défi majeur pour la relève dans la profession, au Canada comme ailleurs ${ }^{19}$.

Les études menées auprès des avocats aboutissent généralement au constat d'une difficulté de conciliation des temps professionnel et familial qui constitue une source de stress et d'insatisfaction générale ${ }^{20}$. Certaines enquêtes montrent que ce conflit peut devenir l'une des principales raisons d'abandon de la profession ${ }^{21}$. Ces enquêtes dressent aussi un autre constat, soit celui des inégalités entre les avocats et les avocates devant cette question de la conciliation comme sur le plan de la carrière en généra ${ }^{22}$. En effet, les femmes déclarent être moins satisfaites que les hommes en la matière ${ }^{23}$. Il apparait également que l'exercice réussi du métier et l'avancement dans la carrière seraient sinon impossibles, du moins apparemment plus difficiles pour les femmes avec enfants. Le statut parental de la femme est par ailleurs un facteur discriminant quant à l'avancement de carrière dans la profession et la situation ne semble pas avoir beaucoup évolué dans le temps. Ainsi, Kay et Gorman ${ }^{24}$ montrent que le conflit travail-famille, les horaires longs et le harcèlement sexuel posent toujours problème pour les femmes avocates, et qu'elles subissent aussi des écarts de salaire, bien que la progression des femmes dans le domaine du notariat et du droit soit importante au Québec, en particulier dans le droit

Structure and Organization, Oxford, Elsevier Science, 2001, 79; Cynthia Epstein, Carol Seron, Bonnie Oglensky, et Robert Saute, The Part-Time Paradox : Time Norms, Professional Life, Family and Gender, New York, Routledge, 1999.

19. Nicky Le Feuvre et Patricia Walters, «Égales en droit? Féminisation de la profession juridique en France et en Grande-Bretagne » (1993) 16 Sociétés contemporaines 41; Elizabeth Sheehy et Sheila McIntyre, dir, Calling for Change: Women, Law, and the Legal Profession, Ottawa, University of Ottawa Press, 2006 ; Canadian Bar Association : Task force on Gender Equality in the Legal Profession, Touchstones for Change : Equality, Diversity and Accountability, Ottawa, Canadian Bar Association, 1993; Heleen FP Ietswaart, "Choices in Context: Life Histories of Women Lawyers in the Netherlands » dans Ulrike Schultz et Gisela Shaw, dir, Women in the World's Legal Professions, Oxford, Hart Publishing, 2003, 353.

20. Ronit Dinovitzer et Bryant G Garth, «Lawyers' Satisfaction in the Process of Structuring Legal Careers » (2007) 41:1 Law and Society Review 1.

21. Joan Brockman, "Leaving the Practice of Law: The Wherefores and the Whys » (1994) 32:1 Alberta Law Review 116; John Hagan et Fiona M Kay, Gender in Practice : A Study of Lawyers' Lives, London, Oxford University Press, 1995; Jean E Wallace, « Can Women in Law Have It All? A Study of Motherhood, Career Satisfaction and Life Balance » (2006) 24 Research in the Sociology of Organizations 283; Jean E Wallace, « Motherhood and Career Commitment to the Legal Profession » (2004) 14 Research in the Sociology of Work 219; Jean E Wallace, " Workto-Non-Work Conflict among Married Male and Female Lawyers » (1999) 20:6 Journal of Organizational Behavior 797.

22. Kenneth G Dau-Schmidt, Marc Galanter, Kausik Mukhopadhaya, et Kathleen E Hull, « Men and Women of the Bar: An Empirical Study of the Impact of Gender on Legal Careers » (2009) 16:1 Michigan Journal of Gender and Law 49.

23. Fiona Kay, «Crossroads to Innovation and Diversity: The Careers of Women Lawyers in Quebec » (2002) 47 McGill Law Journal 699.

24. Fiona Kay et Elizabeth H Gorman, « Women in the Legal Profession » (2008) 4 Annual Review of Law and Social Sciences 299. 
civil $^{25}$. En Ontario, la situation est globalement semblable, et il semble que l'on se préoccupe, là comme au Québec, de la rétention des femmes avocates dans la pratique privée ${ }^{26}$. Certaines auteures ${ }^{27}$ mettent en évidence les normes culturelles ou, dirions-nous, l'éthos professionnel pour expliquer que les femmes soient exclues de ce monde, gouverné par des normes masculines, mais surtout des normes invisibles et bien protégées (《 unwritten » and heavily « guarded» « codes $»)^{28}$.

Si les travaux sont nettement plus nombreux aux États-Unis ${ }^{29}$ et au Canada anglais ${ }^{30}$ qu'au Québec, le Barreau du Québec s'est préoccupé de la question au cours de la dernière décennie, voyant progresser le taux de féminité non seulement dans la profession, mais aussi chez les étudiants en droit. Les femmes sont $60 \%$ aujourd'hui chez les étudiants comme chez les avocats de moins de 10 ans de pratique, la tendance étant donc claire ${ }^{31}$.

Tout en permettant d'entrevoir les points de tension qui caractérisent les parcours des professionnels des deux sexes au sein des professions juridiques, ces enquêtes ne traitent aucunement des modes de gestion de ces tensions. Comment les avocates et les avocats s'organisent-ils pour faire face à leurs responsabilités professionnelles et familiales? Quels choix font-ils au quotidien, et sur l'ensemble de la carrière, pour gérer leur travail, mais aussi leurs responsabilités familiales? Peut-on repérer des stratégies de gestion des temporalités professionnelles et familiales qui aient été éprouvées par les avocats et avocates? Si oui, quelle incidence ont-elles sur les carrières?

L'une de ces thématiques a été abordée dans un sondage du Barreau du Québec/ Cirano $^{32}$, avec une question portant sur l'existence des différents dispositifs et sur la satisfaction à l'égard des mesures de conciliation. Pour ce qui est des différents types d'avantages offerts par les employeurs (Tableau 1), on constate que certains

25. Fiona Kay, « Professional Monopolies and Divisive Practices in Law: "Les Femmes Juridiques” in Civil Law Canada » (2009) 4:3 International Journal of Law in Context 187.

26. Law Society of Upper Canada, Retention of Women in Private Practice: Status Report, Toronto, Law Society of Upper Canada, 2011; Law Society of Upper Canada, Final Report: Retention of Women in Private Practice Working Group, Toronto, Law Society of Upper Canada, 2008.

27. Jean McKenzie Leiper, Bar Codes: Women in the Legal Profession, Vancouver, UBC Press, 2006.

28. Ibid.

29. Gita Z Wilder, Women in the Legal Profession: Findings from the First Wave of the After the JD Study, Washington, NALP, 2007; Joan C Williams, « Legal Professions and Job Demands: Implications for Work/Life Balance » dans Stephen Sweet et Judi Casey, dir, Work and Family Encyclopedia, Chestnut Hill, MA: Sloan Work Family Resolution Network, Boston College, 2007.

30. Kay, supra note 25. Voir aussi : Jean E Wallace, « Motherhood and Career Commitment to the Legal Profession " (2004) 14 Research in the Sociology of Work, 219; Jean E Wallace, "Can Women in Law Have It All? A Study of Motherhood, Career Satisfaction and Life Balance » (2006) 24 Research in the Sociology of Organizations 283.

31. Barreau du Québec, L'avenir de la profession à l'horizon de 2021 : Rapport du Comité sur les problématiques actuelles reliées à la pratique privée et l'avenir de la profession, Montréal, Barreau du Québec, 2011; Barreau du Québec/Cirano, Enquête socio-économique auprès des membres du Barreau du Québec, 2008, Montréal, Barreau du Québec/Cirano, 2009; Barreau du Québec, Women in the Legal Profession, Montréal, Barreau du Québec, 1992.

32. Barreau/Cirano, supra note 11. 
Tableau 1 : Avantages offerts par l'employeur

\begin{tabular}{lll}
\hline Votre employeur vous offre-t-il les bénéfices ou options suivants? & $\begin{array}{l}\text { Femme } \\
\text { \% }\end{array}$ & $\begin{array}{l}\text { Homme } \\
\%\end{array}$ \\
\hline Travail à temps partiel & 30,16 & 30,28 \\
Heures de travail à temps plein flexibles & 59,05 & 59,42 \\
Partage de tâches & 30,90 & 37,31 \\
Congé de maternité non payé & 45,93 & 49,50 \\
Congé de paternité non payé & 42,86 & 49,37 \\
Congé de maternité avec indemnités compensatrices & 66,77 & 62,44 \\
Congé de paternité avec indemnités compensatrices & 59,37 & 59,55 \\
Partenariat à temps partiel & 7,51 & 8,29 \\
Bénéfices de garderies & 11,11 & 10,18 \\
Congés de maladie & 86,24 & 87,19 \\
Invalidité à long terme & 80,95 & 82,29 \\
Formation continue & 82,65 & 82,41 \\
Sabbatique & 48,68 & 50,25 \\
Travail à domicile & 39,05 & 45,60 \\
\hline
\end{tabular}

Source : Barreau du Québec/CIRANO, 2009 : sondage sur 945 répondants féminins et 796 masculins.

sont peu courants, comme le temps partiel, le partage des tâches ou, encore, le partenariat à temps partiel dans un cabinet. Par contre, les indemnités compensatrices pendant le congé de maternité semblent être offertes par une grande partie des employeurs, mais cela n'aide pas la conciliation au jour le jour. Il s'agit ici d'indemnités allant au-delà de ce qu'offre le Régime québécois d'assurance parentale (RQAP), permettant un taux de remplacement plus élevé du revenu ou un congé non payé pour celles qui pourraient ne pas être admissibles au RQAP. Les congés de paternité non payés sont aussi des congés de paternité autres que ceux offerts par le RQAP. Il est aussi possible de jouir d'horaires flexibles, mais il n'est toutefois pas assuré que cette flexibilité soit offerte dans tous les types de milieu de travail et qu'il soit possible d'y avoir recours sans pénalité. C'est pourquoi nous avons voulu compléter ces données quantitatives par une recherche qualitative ${ }^{33}$.

Dans un tel contexte où le soutien organisationnel peut sembler limité, la féminisation de la profession amène le Barreau du Québec (2011) 34 $^{34}$ s'interroger sur le problème de rétention de la main-d'œuvre féminine dans certains types d'exercice comme la pratique privée, principalement dans les grands bureaux qui, comme nous le verrons, peuvent poser problème pour la conciliation. C'est là un enjeu fondamental qui peut mettre en difficulté la relève dans la profession. Comme le note le Barreau ${ }^{35}$ : «le manque d'adaptation de la profession à une

33. La recherche a été menée en partenariat avec le comité conciliation travail-famille du Barreau du Québec, que nous tenons à remercier. Les propos tenus ici n'engagent toutefois que l'auteure.

34. Barreau du Québec, supra note 31.

35. Law Society of Upper Canada (2008), supra note 26. 
réalité qui est loin d'être neutre affecte inévitablement la qualité et la compétence des services juridiques rendus au public. Le départ de femmes de la pratique privée signifie que la profession juridique est en train de perdre une grande partie de ses meilleurs et plus brillants effectifs dans les principaux domaines de pratique $»$.

Il ne faut toutefois pas conclure que ces départs de la pratique privée ne touchent que les femmes, car un bon nombre de jeunes hommes font aussi état de demandes par rapport à la vie familiale, ce qui limite d'autant plus le bassin de la relève. En effet, si les modes d'exercice ne conviennent pas à la vie familiale, pour les femmes et pour de plus en plus de jeunes hommes, comment assurer la relève?

En ce qui concerne les hommes en particulier, les études montrent bien que la priorité accordée à l'emploi lorsqu'on est célibataire peut parfois être renversée lorsqu'arrivent les enfants, comme d'autres chercheurs ont pu le noter dans d'autres milieux, dont la carrière universitaire. Ainsi, Baker ${ }^{36}$ montre que c'est le cas chez les femmes universitaires. Si certaines femmes peuvent alors chercher à séparer davantage les deux sphères du travail et de la famille pour se rendre entièrement disponible pour la famille à la maison, pour les femmes universitaires comme pour les avocates, la carrière est difficile à envisager si l'on priorise la famille. Comme le souligne $\mathrm{Baker}^{37}$, les femmes ont davantage tendance à faire les concessions nécessaires pour concilier travail et famille et elles vont bien sûr en payer le prix sur le plan de la carrière et de leur niveau de stress.

Notre recherche avait donc pour but de vérifier dans quelle mesure la pratique de la profession d'avocat a évolué au fil des ans pour permettre de concilier emploi et famille, et ce, dans les différents milieux de travail et types de pratique.

\section{La méthodologie}

Nous avons choisi de faire une recherche qualitative, par entretiens, afin de mieux comprendre les trajectoires des personnes et les motifs de leurs choix professionnels et familiaux, mais aussi pour compléter les données quantitatives déjà recueillies par le Barreau du Québec/Cirano ${ }^{38}$. Nous avons centré notre questionnement autour de quatre thèmes : l'activité professionnelle et ses contraintes, la vie familiale et son organisation au quotidien, la conciliation emploi-famille ainsi que le recours aux dispositifs de conciliation tels que les congés parentaux, les aménagements du temps de travail, etc. Nous souhaitions mieux comprendre l'influence du genre et de la conciliation sur le parcours professionnel et familial des femmes et des hommes avocats, les choix et les renoncements qu'ils avaient faits, et voir comment certains arrivent à concilier, quelles stratégies ils déploient à cet égard.

\footnotetext{
36. Maureen Baker, « Choice or Constraints: Family Responsibilities, Gender and Academic Career » (2010) 41:1 Journal of Comparative Family Studies 1.

37. Ibid.

38. Barreau du Québec, supra note 31.
} 
Tableau 2 : Les profils des participants selon le sexe et le milieu de pratique

\begin{tabular}{lll}
\hline Milieux & Hommes & Femmes \\
\hline Secteur public/parapublic & 7 & 6 \\
Entreprises (contentieux, etc.) & 3 & 6 \\
Pratique privée : Travail autonome & 2 & 9 \\
Pratique privée : Petits cabinets & 2 & 3 \\
Pratique privée : Moyens/Grands cabinets & 3 & 5 \\
\hline
\end{tabular}

Pour recruter les participants, un appel aux volontaires a été publié sur le site du Barreau du Québec et dans le bulletin diffusé aux membres pour les inviter à nous contacter $^{39}$. Une relance a été effectuée après deux mois pour compléter les profils. Sur 115 avocats qui se sont initialement portés volontaires, nous en avons retenu 46, soit 17 hommes et 29 femmes, en cherchant à couvrir la diversité des milieux de travail. (Tableau $2^{40}$ ).

Nous avons eu quelques difficultés à assurer cette diversité de pratique, principalement à trouver des répondants en pratique privée, et ce, surtout chez les hommes, essentiellement en raison de la forte pression temporelle induite par les contraintes de facturation horaire qui les rend peu disponibles pour des activités non rémunérées. Les personnes de ce milieu ont donc été recrutées grâce à des interventions de membres du Barreau. Pour les hommes volontaires, la plupart d'entre eux s'étaient déjà livrés à une réflexion par rapport à la question de la conciliation travail-famille, et il faut reconnaitre que de ce fait, certains d'entre eux se démarquent des modèles de comportement les plus traditionnels et les plus répandus chez les hommes avocats.

Les entretiens se sont déroulés entre la mi-janvier et la mi-mai 2011 et la plupart ont été réalisés par téléphone ou au moyen de Skype, parce que plusieurs avocats avaient des contraintes temporelles et ne pouvaient se rendre à Montréal, ou alors travaillaient dans une zone mal desservie par les transports publics, d'où la difficulté pour nous d'aller les rencontrer. Presque tous les entretiens ont eu lieu pendant les heures de travail (deux répondants ont préféré être interrogés à domicile et deux répondantes étaient en congé). L'utilisation de ces technologies a permis d'interroger des avocats en régions et de ne pas se limiter aux membres du Barreau travaillant au centre de Montréal. Ainsi, sur 46 répondants, 31 sont basés dans la grande région métropolitaine de Montréal (Longueuil, etc.) et 15 en région.

39. Dans le cadre de cette étude, nous nous intéressons uniquement aux membres du Barreau du Québec.

40. Nous n'avons pas retenu l'ensemble des volontaires puisque plusieurs présentaient des profils semblables (femmes travailleuses autonomes), et après un certain nombre d'entretiens dans ces milieux de pratique (surtout travail autonome, mais aussi secteur public), nous avons jugé avoir atteint le seuil de saturation et nous sommes efforcée d'accroître le nombre d'hommes en pratique privée. 
Les entretiens duraient de $1 \mathrm{~h}$ à $2 \mathrm{~h} 30$, avec une moyenne de $1 \mathrm{~h} 15$. L'accueil des avocats a été très bon, les interlocuteurs étant très intéressés par le sujet et les résultats de l'enquête, ce qui témoigne du fait qu'il s'agit d'un enjeu important au sein de la profession.

La sélection des extraits présentés dans cet article ne signifie pas forcément qu'une majorité d'avocats ont exprimé cet avis (puisque ce n'est pas une recherche quantitative), mais précisons tout de même que c'est ressorti comme un enjeu majeur pour les répondants. Certaines citations, les prénoms et certaines caractéristiques des individus ont été légèrement modifiés pour préserver l'anonymat des répondants.

\section{Les résultats}

Dans cet article, nous avons choisi de nous centrer sur les mesures de conciliation et d'aménagement du temps de travail dont les avocats, hommes et femmes, peuvent se prévaloir pour tenter de concilier emploi et famille, et cela nous a amenée à traiter aussi de leurs stratégies de report ou d'annulation d'activités, ainsi que de la porosité ou la perméabilité des temporalités qu'ils vivent dans l'exercice de leur profession. Nous avons pu observer que, comme la majorité des professionnels, les avocats jouissent d'une certaine autonomie dans l'exercice de leur profession, mais que celle-ci est aussi souvent limitée, en particulier dans certains milieux de pratique, dont les grands cabinets.

Les statistiques (Tableau 2) le montrent bien, les avocats déclarent en grande majorité bénéficier d'un temps plein à heures flexibles. D'ailleurs, cette flexibilité du temps de travail peut être l'un des facteurs explicatifs du niveau de satisfaction que les avocats affichent malgré le nombre important d'heures de travail qu'ils cumulent en regard d'autres professions.

Cette flexibilité temporelle et cette autonomie dans l'organisation de leur activité ont souvent été présentées dans les entretiens comme un avantage considérable de la profession. Le fait d'avoir une charge de travail importante se trouve en partie compensé par la possibilité de s'organiser à sa guise, c'est-à-dire « sans devoir rendre de comptes à personne ». Cette autonomie professionnelle est d'ailleurs vue comme l'une des caractéristiques inhérentes à la profession. Ainsi, si les objectifs sont atteints, on peut s'organiser comme on l'entend et prendre des journées pour ses besoins familiaux, etc.

Tant que je respecte mes objectifs, je suis maittre de ma destinée, je peux prendre une journée de congé ici et là pour pouvoir aller voir ma famille, donc dans la mesure où je suis capable. Si mon travail me permet d'y aller et que mes objectifs sont remplis, je peux y aller n'importe quand, je n'ai pas de permission à demander. (Olivier, 28 ans, marié, deux enfants, petit bureau, région) 
Les professionnels apprécient cette flexibilité, mais elle n'est pas caractéristique de tous les milieux. Dans les organisations des secteurs public, parapublic ou dans les entreprises, cette flexibilité est plus fréquente, bien qu'elle doive être appréciée à l'aune des cultures organisationnelles qui peuvent être plus au moins adaptées et ouvertes à de telles pratiques, alors qu'elle semble clairement plus limitée dans les grands cabinets d'avocats.

\section{Flexibilité des horaires}

Les avocats peuvent aussi, dans certains cas, choisir les moments à consacrer à leur activité professionnelle, quitte à le répartir comme ils le souhaitent sur la journée — et la soirée! — mais aussi sur toute la semaine—et le weekend! Par contre, s'il y a flexibilité des temps de travail, les journées sont souvent longues et parfois lourdes, compte tenu de la charge mentale générée par cette organisation temporelle, mais aussi de la disponibilité requise à certains moments précis pour les clients, ce qui peut se traduire par des discordances temporelles ${ }^{41}$. La journée est ainsi découpée entre le temps familial et le temps de travail, les deux sphères étant souvent imbriquées. Si cela peut convenir aux personnalités dites « intégratrices », qui préfèrent intégrer le travail et les responsabilités personnelles et familiales et n'ont pas de difficulté avec la porosité entre les deux sphères, cela convient généralement moins bien aux personnalités de type " séparatrices », qui préfèrent avoir des cloisons bien étanches entre le travail et la famille ${ }^{42}$.

Notons que la stratégie de report du travail dans les heures théoriquement «privées » ou familiales est fréquemment utilisée pour l'exécution de tâches précises, qui nécessitent de la concentration. Pour une partie des avocats, le temps du bureau n'est pas un temps optimal, car c'est aussi celui de la rencontre avec les clients, le temps des collègues, etc., alors que le temps à domicile est souvent celui où l'on est le moins dérangé (par exemple, lorsque les proches dorment). Ainsi, la flexibilité permet de choisir le temps et les lieux de travail les mieux adaptés, voire de choisir de ne pas travailler s'il y a des priorités du côté de la famille ou simplement si l'on veut prendre du temps pour soi.

En général, je déteste travailler le soir chez moi, je ne suis pas capable. Je préfère aller me coucher plus tôt, me coucher à $9 \mathrm{~h}$ pis de me réveiller à 3 h dans la nuit. Moi, je suis comme ça. Ou tôt le matin pour me concentrer. Souvent, je peux travailler de 4 h à 6 h, pis c'est comme si j'avais fait... si c'est pas le trois quart de ma journée, en vitesse de travail, que

41. Gilbert De Terssac et Diane-Gabrielle Tremblay, dir, Où va le temps de travail?, Toulouse, Éditions Octares, 2000.

42. Diane-Gabrielle Tremblay et Émilie Genin, «Permeability between Work and Non-Work: The Case of IT Self-employed Workers » (2008) 33:4 Canadian Journal of Communication 701. 
je peux accomplir. Tu sais, souvent quand tu dois préparer un dossier, t'as pas de distraction, t'as pas le téléphone, t'as pas de secrétaire qui vient te voir, t'as pas quelqu'un qui arrive sur la soupe. . . Et les fins de semaine j'essaie de moins en moins. En fin de semaine, j'aurais dî. Je m'étais même apporté du travail. Pis j'ai décidé que non. J'ai décidé de prendre du temps pour moi, pis avec les filles. On s'est organisé, pis. . . C'est sûr que le client, je lui avais dit : "ça se peut que je t'appelle ", mais non. Il était pas content, mais bon. Je l'ai fait. C'est ma façon d'avoir un peu de temps à moi aussi, là. Un moment donné, c'est beau de faire l'argent, mais faut aussi prendre du temps pour soi. Je m'aperçois de ça de plus en plus. (Patrick, 33 ans, marié, deux enfants, travailleur autonome, Montréal).

Ces stratégies de réduction du temps de présence au bureau s'accompagnent également d'une remise en question des modalités d'évaluation de la performance basées sur ce temps de présence, le face time. Certains affirment pouvoir être de bons professionnels sans pour autant devoir « faire des heures de fou ». D'autres vont jusqu'à diminuer légèrement leur temps de travail, se jugeant plus efficaces à cause de leur expérience.

La flexibilité peut être échelonnée sur une ou deux semaines, comme le fait cette salariée dans le secteur parapublic, qui a une garde partagée alternée de quatre jours avec son ex-conjoint. Ainsi, les jours où elle a ses enfants, elle part à $4 \mathrm{~h}$ pour les chercher à l'école. Par contre, elle compense largement les jours où c'est son mari qui a les enfants; elle peut alors rester plus tard au bureau pour faire face à la charge de travail, qui reste inchangée.

Par exemple, si un comité se tient jusqu'à 6-7 heures, moi à 4 heures j'ai déjà pris mes bagages et je suis partie chercher mes héritiers! Non, c'est un bon équilibre, il y a des semaines où je rentre plus tôt ou plus tard, parce que je n'ai pas mes enfants tout le temps, j'ai la garde partagée, donc ça me permet de pouvoir bien doser de telle sorte que. . . il se peut qu'une semaine ma présence en termes d'heures puisse être moins grande ici, par contre la semaine d'après ça va être plus. (Françoise, 41 ans, divorcée, deux enfants, secteur parapublic, Montréal)

Toutefois, dans d'autres organisations, cette flexibilité se trouve limitée, car les professionnels sont confrontés à des exigences de disponibilité pour des clients internes ou externes. Ainsi, les heures de bureau sont celles où ils reçoivent les appels et les mandats, sans pouvoir choisir les moments de ces appels. La notion de service au client exige qu'ils s'en tiennent aux horaires classiques de bureau. 


\section{Limites et revers de l'autonomie professionnelle}

Pour une grande partie des avocats rencontrés, la flexibilité des horaires et de l'organisation du travail est un avantage non négligeable du fait d'être des professionnels. Par contre, les contraintes de l'activité, ainsi que les objectifs à atteindre et le face time (présence au bureau) semblent parfois réduire l'ampleur de cette autonomie. Alors qu'une partie des avocats rencontrés affirment jouir d'une certaine flexibilité en ce qui a trait à leur temps de présence au bureau, ils ne sont toutefois pas libérés de l'obligation d'un temps de présence au bureau qui tend à s'inscrire dans un cadre proche des horaires classiques (9 à 5).

En fait, je peux rentrer quand je veux, je peux partir quand je veux, mais les associés s'attendent quand même que de 9 h à 17 h on soit là. Quand même un minimum, mais ils n'exigent pas. (Laure, 33 ans, en couple, un enfant, petit bureau, Montréal)

Ainsi, les objectifs de performance ou de travail sont connus, et les avocats doivent planifier leurs activités au quotidien, mais aussi la performance sur l'ensemble de l'année. Cette contrainte est d'autant plus forte pour les travailleurs autonomes, dont les revenus d'activité dépendent directement du volume de clients. En effet, dans ce contexte, ils doivent créer et entretenir leur clientèle, tenter de la développer, en se rendant disponible pour les clients, selon leurs besoins. Il arrive aussi qu'au lieu de donner un sentiment de maîtrise de son temps, la gestion de cette flexibilité sous contrainte induise des effets psychologiques et physiologiques qui se traduisent par une réelle fatigue et des problèmes de santé. Certaines avocates ont évoqué qu'elles ne dormaient pas assez, qu'elles étaient fatiguées tous les matins, ne se réveillaient jamais en forme. Et le weekend, la vie familiale prend le dessus avec d'autres horaires :

Et les fins de semaine aussi, on me réveille aussi parce que les enfants ont la natation. (Danny, 42 ans, mariée, trois enfants, entreprise, Montréal)

Cela peut parfois imposer des horaires très stricts et une forte discipline dans l'organisation de sa vie sociale, un avocat ayant même indiqué qu'il considérait nécessaire de ne pas sortir en semaine, de ne pas boire, d'éviter tous les excès pour s'assurer de bien dormir.

Moi je ne sors pas, je ne bois pas, j'évite les excès, parce que justement, j'en ai pour trois jours, parce que j'aurai un sommeil beaucoup moins réparateur. Certains diraient que c'est une vie plate, je ne suis pas d'accord avec ça (Nicolas, 27 ans, marié, trois enfants, travailleur autonome, région)

Les témoignages de plusieurs avocats nous indiquent qu'ils ont de la difficulté à assumer la charge mentale et physique. Ils disent « être lessivés » à la fin de ces 
journées où l'on n'arrive jamais à joindre les deux bouts et où la difficile conciliation travail-famille n'est pas vraiment réussie. Plusieurs vivent également un sentiment de culpabilité, ne se sentant jamais assez performants, ni au travail ni à la maison, comme en témoigne l'extrait suivant :

En ce moment, ma plus grande contrainte c'est d'être disponible pour mon employeur et être disponible pour mes enfants. Trouver un équilibre entre les deux. Mais il arrive un moment où l'on se sent coupable, on se sent coupable devant l'employeur parce qu'on dit " je m'excuse je dois aller chercher les héritiers », mais aussi devant les enfants, parce que c'est souvent " maman ne peut pas, maman doit aller au boulot, peut pas faire ci ou ça». On se sent toujours coupable, il faut apprendre à vivre avec mais j'essaie de faire de mon mieux pour équilibrer les deux. Et être avec mon amoureux (Françoise, 41 ans, divorcée, deux enfants, secteur parapublic, Montréal)

\section{Les aménagements du temps de travail}

Les possibilités d'aménagement du temps de travail diffèrent beaucoup d'un milieu à l'autre. Alors que les avocates indiquent qu'une réduction du temps de travail serait l'une des solutions à leurs difficultés de conciliation, elles ne sont pas nombreuses à y avoir accès. Le secteur public semble offrir plus de possibilités sur ce plan, mais cela dépend également du cadre organisationnel et des arrangements locaux. Un avocat, conseiller dans un ministère et père de trois enfants, a demandé une semaine de 32 heures pour pouvoir bénéficier de quelques jours de congé de plus. Ces journées lui permettent de faire face aux urgences familiales (sa femme est médecin et les possibilités pour elle de quitter son travail au cours de la journée sont plus limitées).

Dans les entreprises privées, la culture organisationnelle déterminera dans une grande mesure l'accès aux dispositifs de réduction du temps de travail. Le fait que les professionnels ne soient pas syndiqués les place dans une situation délicate de ce point de vue et plusieurs se sont vu refuser l'aménagement de leur temps de travail. Nous avons noté toutefois que certains grands bureaux commencent à accepter des assouplissements.

Une avocate pouvait prendre son lundi pendant trois mois après son congé de maternité d'un an, car elle avait accumulé un nombre de jours de congé important et avait décidé d'étaler son crédit temps sur plusieurs mois. Toutefois, elle indique que son congé avait coïncidé avec une baisse d'activité liée à la crise et que, dans ce contexte, l'employeur avait été plus ouvert à accepter ses absences.

La possibilité de passer à une semaine de quatre jours a été la mesure citée le plus souvent par les personnes lors des entretiens (surtout les parents d'enfants en bas âge), hommes ou femmes, comme solution optimale pour les aider à atteindre leur objectif de conciliation. Toutefois, elle n'est envisageable que dans 
très peu de cas, car le milieu et le travail en lui-même ne s'y prêtent apparemment pas, même si plusieurs le souhaitent. En effet, même les milieux réputés plus ouverts à la conciliation, comme le secteur public, ne permettent pas systématiquement d'aménager son temps de travail ou de travailler quatre jours par semaine. $\mathrm{Au}$ mieux, les avocates peuvent songer à un retour progressif au travail, mais d'après ce que nous avons vu, ce sont des situations qui ne sont pas généralisées, auxquelles on n'a pas droit automatiquement. Cela peut aussi avoir un effet sur le plan de carrière par la suite, notamment en ce qui concerne l'accès au statut d'associé dans un cabinet, qui peut alors être difficile, voire impossible, d'accès.

Les avocats tentent donc de rendre leur vie et surtout la conciliation travailfamille plus soutenable. Ainsi, certains ont tenté de négocier l'étalement des congés annuels personnels non utilisés pour combiner les congés non utilisés aux congés parentaux et avoir ainsi une semaine de quatre jours pendant un certain temps. Pour d'autres, notamment les travailleurs autonomes, ce sont des conditions qui peuvent se négocier avec le client : dans ce cas, on fixe à l'avance les journées correspondant à leur disponibilité.

Notons qu'une avocate seulement a pu bénéficier d'un temps partiel au sein d'une organisation dans le secteur public, mais en fait, cela s'apparentait davantage à un temps partiel « subi ».

\section{Les stratégies d'organisation temporelle au service de la conciliation emploi-famille}

Alors que les différents dispositifs formels de réduction du temps de travail ne sont pas fréquemment utilisés dans la profession, le temps plein à heures flexibles (Tableau 2) semble être accessible à un plus grand nombre. C'est d'ailleurs l'un des éléments qui ressort des entretiens avec les avocats dans les différents milieux : ce qui permet de compenser un investissement temporel important, c'est la flexibilité des horaires et l'autonomie dont jouissent les avocats pour les organiser. Toutefois, cette flexibilité donne lieu à un ensemble de stratégies très particulières. Les efforts de planification et d'organisation sont très importants, en particulier du côté des femmes. Certaines parlent d'une «torsion » des normes habituelles de travail qu'il faut effectuer pour arriver à concilier travail et famille. Quelles sont donc les stratégies de gestion des temporalités professionnelles et familiales utilisées par les avocats? Il nous semble que c'est ici un des apports les plus importants et originaux de notre recherche puisque si l'on s'est beaucoup intéressé dans les recherches aux situations des femmes qui quittent des postes professionnels et aux motifs qui les incitent à quitter - le fameux « opting-out $»^{43}$ - il n'existe pas, à notre connaissance, de travaux traitant des stratégies utilisées par les femmes

43. Supra note 15. 
avocates pour réussir à concilier travail et famille, au-delà de la flexibilité des horaires de travail ${ }^{44}$.

Ces stratégies sont pourtant fort intéressantes et permettent aux avocates de gérer leur disponibilité relative au temps professionnel tout en tenant compte des besoins de la famille. Ce qui est le plus apprécié, c'est la flexibilité dans la gestion de son temps professionnel, une flexibilité qui a toutefois un prix non négligeable, notamment en raison de la charge mentale qu'elle induit. Voyons d'un peu plus près ces stratégies qui peuvent se résumer ainsi : planification, intensification et report d'activités.

\section{Stratégie d'intensification du travail}

Interrogés sur les modalités concrètes de leur organisation temporelle au travail, plusieurs avocats ont souligné que le fait d'avoir des enfants les a obligés à modifier la gestion de leur travail, essentiellement en planifiant davantage leur travail et les diverses activités, mais surtout en intensifiant leurs heures de travail. Puisqu'il ne leur est plus possible de travailler tard le soir ou la fin de semaine, les obligations familiales limitant désormais cette option, surtout en l'absence de soutien de la part du conjoint, ils réduisent les heures de présence au bureau, mais prennent moins de pauses, et évitent les repas d'affaires le midi.

L'intensification s'impose notamment en raison des contraintes des heures des garderies. Cette nouvelle régulation temporelle est parfois appréciée par les avocats qui trouvent ici la « bonne excuse » pour ne pas prolonger leurs heures au bureau. Par contre, certains d'entre eux reprennent le travail une fois les enfants couchés. Plusieurs soulignent qu'ils retrouvent un rythme de travail plus « vivable » même si, à la longue, avec la pression des objectifs, cela peut leur rendre la vie beaucoup plus compliquée.

L'une des stratégies auxquelles les avocats recourent consiste à maximiser le rendement du temps de présence au bureau. Cette stratégie de densification du travail peut se déployer de différentes façons : organiser les rendez-vous au plus serré dans l'agenda, éviter les pauses et les repas à l'extérieur pour travailler les dossiers, limiter les interruptions dans le travail par les appels ou les visites. Certains avocats, par exemple, n'hésitent pas à se servir de leur messagerie téléphonique et à rappeler plus tard en fonction des urgences.

Cette intensification passe souvent par la suppression des temps " personnels », c'est-à-dire des pauses, voire de la pause pour le lunch; la plupart des avocates ont dit manger leur sandwich au bureau. Elles ont ainsi le sentiment d'une plus grande efficacité dans le travail et l'utilisation de leur temps.

44. Scott Schieman et Paul Glavin, « Trouble at the Border? Gender, Flexibility at Work, and the Work-Home Interface » (2008) 55:4 Social Problems 590. 
Quand je suis là, j'ai l'impression d'être encore plus efficace, plus focusée parce que je me dis, je ne peux plus rester. Donc au niveau des heures facturables à date, là, j'arrive à rentrer dans les objectifs. Donc dans les heures je travaille peut-être moins, mais j'ai l'impression d'être plus efficace. Je coupe aussi dans ma pause de diner. Je me disais avant que je pouvais partir le soir, mais là il faut que je quitte parce qu'il faut m'occuper de mon enfant puis le souper, etc. (Laure, 33 ans, en couple, un enfant, petit bureau, Montréal)

Les femmes se démarquent de leurs confrères sous certains angles. Ainsi, elles s'investissent beaucoup plus dans les activités de planification, elles rationalisent et se fixent des agendas où chaque minute est chronométrée. Le prix à payer pour cette efficacité et le sentiment de maîtrise du temps qui l'accompagne est « l'impression d'être sous un horaire tout le temps ", comme l'illustre l'extrait suivant.

Je suis organisée dans ma vie, je suis très organisée au travail, quand je suis au travail, je suis au travail, quand je travaille je me concentre, je suis une fille qui travaille vite, qui travaille bien et pourtant mon travail a toujours une belle qualité. J'ai vu les prédécesseurs faire, les gars, qui étaient très vendeurs, des VP typiques qui perdaient beaucoup de temps dans leurs journées, des lunchs qui n'en finissent plus, et moi je m'organise pour avoir pas plus de deux lunchs par semaine, parce que ça prend trop de temps. Donc j'ai beaucoup plus tendance à manger mon sandwich en même temps que je travaille pour qu'à 4 h 30 ce qui était dîu, mes livrables pour la journée, c'est fait. Puis je m'organise, je planifie, je sais toujours ce que j'ai à faire, dans quel ordre, je pense que c'est ça la clé, c'est la seule façon, sauf que j'ai toujours l'impression d'être sous un horaire tout le temps, tout le temps, c'est sûr que ça a un côté qui est moins fun. (Danny, 42 ans, mariée, trois enfants, entreprise, Montréal)

Les hommes que nous avons interrogés n'ont pas vraiment mentionné de telles stratégies de planification et de gestion au plus près de leur agenda, et lorsqu'ils parlent de l'efficacité qui leur permet de faire leur travail plus vite, ce n'est pas présenté en termes de moyens pour optimiser la conciliation travail-famille, mais plutôt comme une conséquence de leur expérience professionnelle, un moyen d'assurer une meilleure performance au travail essentiellement.

\section{Report ou suppression de certaines activités}

La recherche d'une disponibilité maximum pour la famille pendant les soirées conduit les avocats à revoir un peu le déroulement de leurs activités habituelles. 
Si c'est plus souvent le cas des femmes, certains pères ont aussi revu leur emploi du temps. Ainsi, Nicolas a choisi de ne plus prendre de rendez-vous en soirée, sauf en cas d'urgence, quitte à recevoir les clients en fin de semaine, pendant l'heure de sieste des enfants. Cela lui permet de compenser l'un des principaux « défauts » de son activité : l'imprévisibilité.

Avant, je faisais en soirée, avant que j'aie des enfants, et depuis je ne le fais plus, parce que je veux être là pour aider ma conjointe pour le souper et le coucher, donc je le fais le midi ou en fin de semaine. Un samedi sur deux, je donne des rendez-vous entre une heure et quatre heures parce que c'est la sieste des enfants. Donc il y a eu un changement là, toujours dans le but de la conciliation, toujours dans le but de maximiser mon temps à la maison. Donc il y a eu un changement des heures des rendez-vous et $d u$ travail au bureau. (Nicolas, 27 ans, marié, trois enfants, travailleur autonome, région)

Depuis la naissance de son enfant, non seulement Fabienne ne participe plus aux activités sociales le soir, mais elle ne fixe plus de rendez-vous après les heures de bureau. Elle préfère rentabiliser son temps de présence, notamment en coupant dans sa pause de midi.

Je dois faire un peu comme une ligne entre ma pratique depuis que je suis de retour de congé de maternité . . Alors, actuellement, disons que je n'accepte plus beaucoup de représentation le soir. C'est plutôt rare alors qu'avant, j'étais beaucoup sollicitée pour des cocktails, des soirées ou des trucs avec les clients. Mes représentations, j'essaie de les faire sur l'heure du dîner par exemple, ou de fixer des rendez-vous qui sont des trucs où je peux pas facturer. Souvent sur l'heure du dîner puis le soir, c'est beaucoup plus limité. (Fabienne, 38 ans, mariée, un enfant, grand bureau, Montréal)

Les parents (de jeunes enfants surtout) cherchent également à réduire les déplacements à leur strict minimum. Néanmoins, ces activités peuvent être maintenues à condition que les familles puissent y participer. Certains avocats exerçant comme travailleur autonome intègrent parfois la famille à leurs activités professionnelles et ils jugent les réactions des clients plutôt positives, ou tout au moins, ne se sentent pas jugés pour cette raison. Ainsi, Jean est engagé dans des activités politiques et il amenait sa conjointe avec leur enfant à ses réunions en dehors de la ville, profitant ainsi du congé de maternité de sa conjointe.

L'avantage c'était qu'après la naissance de ma fille, ma conjointe était en congé maternité, donc ça veut dire qu'on avait une bonne souplesse à ce niveau-là. Puis, par exemple, l'avantage est que ce sont des réunions de 
jour et souvent par exemple, c'était en dehors de la ville, ma conjointe venait avec moi et avec le bébé. Donc ma fille a vécu cette année avec nous, mes collègues étaient très gentils là-dessus. (Jean, 36 ans, marié, un enfant, associé, grand bureau, Montréal)

Il faut dire que pendant la période où la femme est en congé de maternité, et justement parce qu'elle prend l'ensemble du congé parental (peu de pères avocats en prennent une grande partie), les avocats peuvent se trouver dans une situation apparentée à celle des anciennes générations, avec une femme qui prend en charge tout le travail domestique et familial. Mais cela change généralement au terme du congé de maternité-parental. De plus, la suppression de certaines activités peut pénaliser les avocats quand il s'agit d'activités qui peuvent servir à développer la clientèle et à améliorer ainsi leur évaluation à la fin de l'année. Finalement, les avocats parents qui ne peuvent ou ne veulent pas transférer entièrement les activités parentales à leurs conjoints se trouvent de ce fait davantage pénalisés que les autres.

Ajoutons qu'il existe des activités dans le travail des avocats qui ne peuvent être reportées, comme les dates de procès et le temps de présence à la cour. Ainsi, les stratégies de report ou de choix des temps optimaux pour la réalisation de certaines tâches butent sur ces moments qui exigent une disponibilité sans changement possible. Les procès à la cour sont fixés longtemps d'avance, et leur report n'est pas bien vu, ni par les juges ni par les clients. Il faut alors trouver une autre solution.

Le contexte de concurrence place également les avocats face à un dilemme : risquer de perdre certains clients, disponibles le soir uniquement, ou être moins disponible pour les enfants et les activités domestiques.

\section{Une grande porosité des temps}

Une observation intéressante que nous avons faite chez les avocats, c'est la très forte porosité ou interpénétration des temps professionnels et familiaux, que l'on pourrait apparenter à la disparition des frontières entre les deux mondes ${ }^{45}$. Ce phénomène peut s'analyser comme l'un des revers de la flexibilité professionnelle. En effet, les avocats sont nombreux à affirmer pouvoir s'occuper de leurs affaires personnelles pendant les heures de bureau. Par contre, le domicile familial peut être envahi par le travail à différents moments et sous différentes formes. Ainsi, dans la profession juridique, la frontière entre la vie professionnelle et la vie familiale n'est pas très étanche. On observe un haut degré d'acceptation des phénomènes de débordement entre la sphère professionnelle et la sphère familiale. Ce phénomène n'est cependant pas propre aux avocats, car pour « pour la plupart des parents travailleurs, articuler travail et famille n'est guère aisé et nécessite un jonglage ou

45. En lien avec la « Border theory » voir ibid. 
une "débrouille" " ${ }^{46}$ et c'est particulièrement le cas dans le secteur informatique (Tremblay et Genin, 2008). C'est justement dans ces moments de jonglerie entre les activités professionnelles, parentales et domestiques que la question de l'inégalité des sexes émerge clairement.

Le développement de nouvelles technologies comme le téléphone intelligent (Blackberry), la croissance de la taille des organisations et surtout la plus grande concurrence entre les bureaux d'avocats sont autant de facteurs cités pour expliquer l'intensification du travail, comme la plus grande porosité des sphères du travail et de la vie personnelle/familiale.

Comme d'autres professionnels vivant le même type de situation, les avocats acceptent généralement très bien ces débordements et les perçoivent comme étant la contrepartie presque naturelle des conditions avantageuses que leur offre leur profession en matière de salaire et de flexibilité temporelle. Danny en parle très bien, en disant ne plus savoir où mettre la limite entre la sphère familiale et la sphère professionnelle, car ces activités sont liées et peuvent prendre place l'une comme l'autre à toute heure. Cela n'est pas sans créer de la confusion dans la gestion des deux sphères pour les personnes qui préféreraient les séparer. D'autres personnes par contre, celles que l'on qualifie d'intégratrices plutôt que de séparatrices, peuvent préférer intégrer les deux sphères pour avoir le sentiment de mieux maîtriser leur temps et leur charge de travail globale ${ }^{47}$.

C'est difficile de départager les deux sphères. Moi, je me rends compte que quand je suis au travail, mes enfants c'est tout le temps, là " maman j'ai ci, maman j'ai ça, on a fini l'école peux-tu venir me chercher? 》ou " j'ai rendez-vous chez l'orthodontiste ". . . Je me pose beaucoup de questions là-dessus, j'habite à quelques minutes d'ici, mes enfants vont à l'école aussi dans le coin, donc si on a un rendez-vous chez l'orthodontiste, c'est facile, le rendez-vous est à 3 heures et demi, je les ramasse à l'école à $3 h$, ou à $3 h 15$, je les ramène chez le médecin et des fois je peux revenir au bureau. Donc à un moment donné on dirait que tout se mêle, les enfants, le travail, la maison, les enfants c'est les heures de travail, le travail c'est les heures des enfants, parce que, aussi, je veux dire, à 4 h 30 quand j'ai quitté le bureau mes employés sont encore là, et même si je rentre chez moi, je continue à recevoir les courriels et oui quand je suis avec les enfants, je les regarde, je vais répondre, les enfants disent " dépose ton Blackberry, je te parle »... C'est vrai, je trouve ça très difficile ce boulot parce que c'est comme s'il n'y a plus de cette démarcation, comme si toute la vie c'est la famille et puis toute la vie c'est le travail en même temps. On n'arrive plus

\footnotetext{
46. Bernard Fusulier, David Laloy et Émilie Sanchez «Être au service et articuler travail/famille » (2009) 154 Informations sociales 22.

47. Tremblay et Genin, supra note 42 .
} 
à compartimenter et je trouve ça très difficile. (Danny, 42 ans, mariée, trois enfants, entreprise, Montréal)

N'empêche que cette porosité, même si elle est porteuse de confusion entre les temps et les espaces, l'un envahissant l'autre, représente aussi une certaine maitrise du temps. La citation plus haut illustre de manière extrême cette porosité mais ce cas n'est pas unique, et plusieurs avocats et avocates nous ont fait part de ce type d'expérience. En effet, qui va s'étonner du récit d'un avocat qui dit préparer ses dossiers à la maison la veille d'un procès? Cette disponibilité que les avocats essaient d'aménager pour le travail et pour la famille peut conduire certains à ne plus pouvoir décrocher.

Le fait de travailler à la maison peut également être recherché parce qu'il permet de travailler en toute tranquillité, sans être dérangé, une condition difficile à atteindre au bureau. Par ailleurs, les moments de travail réalisé au domicile peuvent être choisis au mieux pour ne pas empiéter sur les temps communs des conjoints. Ainsi, le travail le soir n'est pas rare mais se fait généralement lorsque le conjoint est absent, afin de favoriser le temps familial.

Lorsque j'ai du travail, j'essaie de le faire lorsqu'elle n'est pas à la maison, pour ne pas le faire lorsqu'elle est là. Et ce que je fais aussi . . . si j'ai une urgence à faire, je vais plutôt me lever très tôt le matin, à $5 \mathrm{~h} d u$ matin. Je suis plus efficace, je suis tout seul, les enfants dorment, ma femme dort . . . c'est plutôt d'avoir du temps sans interruption. C'est pour ça aussi, travailler au bureau ça va mal, le téléphone sonne, il y a des gens . . . le samedi ça va bien, tôt le matin aussi, personne n'appelle. (Nicolas, 27 ans, marié, trois enfants, travailleur autonome, région)

D'autres, au contraire, essaient tant bien que mal de délimiter clairement les deux sphères et de ne pas laisser l'une envahir l'autre. Certains disent ne pas être aussi efficaces lorsqu'ils travaillent à domicile, mais face aux urgences et aux demandes pressantes de certains clients, on est bien obligé d'allumer son ordinateur le soir pour répondre.

Quand je travaille jusqu'à 6 ou 7 h du soir, je ne retravaillerai pas à la maison après. Mais y arrive des situations, comme hier par exemple je suis arrivée à la maison vers 7 h et à 7 h 30, j'avais un appel puis le client désirait avoir une opinion le soir même, alors j'avais amené ma documentation pis finalement, j'ai soupé, j'ai couché bébé pis je me suis réinstallée pour une heure pour rédiger une brève opinion pis j'envoie ça au client . . . (Fabienne, 38 ans, mariée, un enfant, grand bureau, Montréal)

L'exemple de Fabienne montre cette tension qui peut caractériser la situation des parents avocats. Cependant, les exigences réelles du travail, incarnées par la figure 
du client, incitent souvent les avocats à transgresser les règles qu'ils se sont fixées, par exemple de ne pas apporter du travail à la maison ou de ne pas répondre lorsqu'ils téléphonent. Cette exigence de disponibilité pour les clients semble s'imposer très fortement à un grand nombre d'avocats. Si elle n'est pas toujours vécue sur un mode négatif, en raison de l'importance de la notion de service au client, qu'il soit institutionnel ou particulier, elle est pour ainsi dire presque « sacrée » dans la profession; en effet, c'est le service rendu - et de préférence de bonne qualité - qui permet de produire les rentrées d'argent attendues . . . et éventuellement d'accéder au statut d'associé. Cette importance du service au client peut être vue comme un des éléments centraux de l'éthos professionnel des avocats et la figure du client vient en quelque sorte s'imposer entre l'employeur et l'avocat, et imposer les dépassements d'horaires dans de nombreux cas. L'employeur n'a pas nécessairement à imposer cette obligation de service, puisque l'éthos et les normes professionnelles du milieu le rendent incontournable de toute manière. Si plusieurs avocats reconnaissent que lesdites « urgences » ne sont pas toujours si urgentes, ils ne seront pas le premier à briser la loi de « l'obligation de répondre en tout temps au client », surtout si l'employeur paie le téléphone intelligent (Blackberry).

Il faut ajouter que l'arrivée des enfants tend à accroître la division traditionnelle $\mathrm{du}$ travail domestique entre les hommes et les femmes et à renforcer le rôle des femmes, même si nous avons observé un bon nombre de couples relativement égalitaires sur ce plan. On pourrait dire qu'il y a davantage d'hommes profitant d'une situation de couple traditionnel (homme investi dans la profession, femme davantage dans la famille) dans les grands bureaux, alors que dans le secteur public ou les contentieux, les hommes avocats peuvent se permettre de s'investir davantage dans la famille. Si les salaires des avocats pouvaient permettre de déléguer une bonne partie des tâches domestiques, nous avons noté que peu d'entre eux déléguaient les tâches centrales comme les repas, les courses ou les soins aux enfants. Seules les activités de ménage tendent à être externalisées. La plupart placent les enfants en services de garde, plutôt que d'avoir une nounou à domicile, et en cas de difficulté, on aura éventuellement recours aux grands-parents. Certains couples se sont même rapprochés de leurs parents pour pouvoir profiter de ce service en cas de besoin.

On observe donc tout de même différents modèles de couples, soit les couples traditionnels (homme investi dans la profession, femme davantage dans la famille), les couples égalitaires (les deux se partageant les rôles) et quelques rares cas d'inversion, où l'avocat est plus présent auprès de la famille, parce que sa femme a un emploi plus exigeant et généralement plus rémunérateur; elle est médecin et lui avocat dans le secteur public par exemple. Chacun de ces types développe des stratégies de conciliation différentes à l'image de ce qu'ont observé d'autres études ${ }^{48}$.

48. Phyllis Moen et Yan Yu, « Effective Work/Life Strategies: Working Couples, Work Conditions, Gender, and Life Quality » (2000) 47:3 Social Problems 291; Youngjoo Chaa, " Reinforcing Separate Spheres: The Effect of Spousal Overwork on Men's and Women's Employment in DualEarner Households » (2010) 75:2 American Sociological Review 303. 
Il est clair que le fait d'obtenir plus de flexibilité d'aménagement de son temps de travail incite à rester chez un employeur, une hypothèse qui a été testée dans d'autres travaux ${ }^{49}$. Par contre, les avocats et surtout les avocates qui n'obtiennent pas cette flexibilité seront très tentés de s'établir à leur compte, d'ouvrir un bureau chez elles, comme travailleuses autonomes.

\section{Conclusion}

Nous avons pu le constater, les avocats bénéficient souvent d'une grande autonomie et d'une bonne flexibilité dans l'organisation de leur temps de travail, comme c'est le cas pour bon nombre de professionnels. Ils peuvent ainsi s'organiser pour décaler des activités, les reporter le soir, le weekend ou très tôt le matin, mais par contre, ils nous disent tous, et surtout dans les grands cabinets, que les patrons aiment les voir au bureau dans les heures «normales » de travail, soit de $9 \mathrm{~h}$ à $17 \mathrm{~h}$. La flexibilité s'en trouve donc quelque peu limitée.

Néanmoins, lorsqu'ils ont des enfants et doivent concilier le travail avec la vie familiale, les avocats développent des stratégies pour « faire les heures facturables », mieux performer, sans trop déborder sur leur temps personnel ou familial. Dans certains cas, cela se présente sous forme de report d'activités, mais dans d'autres il y a aussi intensification du travail au bureau, élimination de la pause de dîner, recours au répondeur, suppression des pauses café, des échanges avec les collègues, etc. Enfin, alors que dans certains cas les activités sociales en soirée (cocktails, etc.) sont essentielles pour développer la clientèle, certaines avocates affirment avoir éliminé ces activités, tout à fait incompatibles avec la famille; mais de fait, cela en amène plusieurs à changer de milieu de travail, à se déplacer d'un grand cabinet, où cela est quasi obligatoire, vers un plus petit bureau, ou vers le secteur public.

Certaines activités ne peuvent toutefois pas être déplacées, comme les procès, et c'est là une contrainte que vivent certains avocats. Certains peuvent tenter de fixer les horaires en fonction de leurs obligations familiales (déplacements à la garderie ou à l'école pour y déposer et reprendre les enfants), mais cela n'est pas toujours facile.

La mise en évidence de ces stratégies (planification, intensification et report) nous semble constituer un des apports majeurs de cette recherche ${ }^{50}$ et il serait sans doute intéressant que les entreprises facilitent la mise en œuvre de ces stratégies et soutiennent les femmes qui les utilisent. Le report d'activités n'est pas toujours facile, mais dans les faits, les avocats et leurs employeurs peuvent parfois admettre que ce qui est dit urgent ne l'est pas toujours autant. Le rôle du client dans l'accentuation du caractère d'urgence pourrait sans doute être analysé et

\footnotetext{
49. Phyllis Moen, Erin L Kelly, et Rachelle Hill, « Does Enhancing Work-Time Control and Flexibility Reduce Turnover? A Naturally Occurring Experiment » (2001) 58:1 Social Problems 69.

50. Merci à l'évaluatrice externe qui l'a souligné.
} 
remis en question dans certains cas afin d'améliorer la qualité de vie des avocates et avocats et, ce faisant, d'accroître leur satisfaction en emploi et de réduire les départs vers d'autres milieux de travail (des grands bureaux vers les petits ou le travail autonome). Il ne semble pas y avoir de recette magique permettant de concilier la famille avec un poste d'avocat, surtout dans les grands cabinets, mais on peut s'en tirer avec diverses stratégies d'aménagement temporel.

Enfin, nous avons pu observer qu'il existe deux types de personnalité en ce qui concerne ces ajustements, soit les personnes qui préfèrent intégrer les activités de travail et celles liées à la famille, pour avoir l'impression de mieux performer sur les deux plans, et les autres, qui préfèrent au contraire les séparer, pour ne pas subir l'envahissement d'une sphère par l'autre. On pourrait toutefois penser que ce ne sont pas seulement des traits de personnalité, mais que ces stratégies peuvent être plus faciles ou carrément impossibles selon le milieu de travail ou le type de client. De ce fait, si une avocate souhaite absolument séparer les deux sphères de vie, et que les clients organisationnels exigent des réponses rapides et ne le permettent donc pas, elle aura tendance à quitter pour un autre milieu (secteur public ou contentieux d'une entreprise) ou à choisir le travail autonome pour satisfaire ce souhait.

Par ailleurs, il est généralement plus facile pour les hommes de séparer les deux sphères, car il est plus fréquent qu'ils soient moins investis dans la vie familiale. Par contre, les jeunes avocats qui sont fortement investis dans la sphère familiale ont autant de difficulté que les femmes à ne pas se laisser envahir par les activités professionnelles à la maison; s'ils sont dans un grand bureau, il n'est pas rare qu'ils reprennent le travail en soirée ou au petit matin, comme nous l'avons vu plus haut. Il est intéressant de voir qu'il n'y a pas que le supérieur immédiat ou le patron qui exerce des pressions sur les salariés ou les associés, la figure du client étant particulièrement dominante dans cette profession. En effet, on voit apparaître ici une figure qui n'est pas toujours présente dans la relation salariale, soit celle du client, et qui peut obliger les avocats à transgresser les règles et les priorités qu'ils se fixent en matière de conciliation emploi-famille et, surtout, de préservation de certains espaces-temps pour la vie privée et familiale. En effet, nos entrevues ont montré que pour un grand nombre d'avocats et d'avocates, l'exigence de disponibilité pour les clients s'impose sans possibilité de négociation, et ce, même si les appels ou les messages prétendument « urgents » ne le sont pas toujours à leurs yeux. Cependant, pour ne pas perdre le client, on doit accepter cette interférence dans son temps personnel ou familial (en soirée, en weekend, et parfois même en vacances. . .), car il serait très mal vu de ne pas être disponible alors que les autres avocats le sont. Ainsi, cette pression est vue comme une contrainte imposée à tous, mais il en est ainsi justement parce que tous acceptent de plier face à la figure du client, qui s'interpose dans la relation d'emploi.

Il faut reconnaître que cette interférence n'est pas toujours vécue négativement par les avocats, essentiellement parce qu'elle semble intériorisée par tous, incontournable, parce que les activités juridiques sont pour beaucoup des activités de 
service à la clientèle. De ce fait, la notion de client, institutionnel ou particulier, paraît " sacrée » dans la profession et tous reconnaissent que c'est le service rendu et le service de bonne qualité qui permet de générer les profits attendus dans les grands cabinets notamment, et d'avoir accès à une promotion ou au statut d'associé.

Dans une perspective de plus long terme, on peut aussi s'interroger sur l'effet de ces stratégies d'aménagement du temps de travail (intensification, report) tout comme de la prise de longs congés parentaux, sur les carrières. Comme les hommes ne prennent que de courts congés (en moyenne 7 semaines, contre 10-11 mois pour les femmes), même lorsqu'ils prennent le congé de paternité qui leur est réservé et non transférable à la conjointe dans le cadre du Régime québécois de congé parental, ils vivent moins d'impacts négatifs à long terme que les femmes, qui peuvent perdre des dossiers, des clients, voire des promotions dans l'organisation. La prise d'un long congé parental est coûteuse pour les femmes avocates, peut-être encore davantage que dans les autres milieux ${ }^{51}$. Elle est coûteuse sur le plan du salaire comme l'ont montré d'autres travaux ${ }^{52}$, mais aussi sur le plan de la progression professionnelle et parfois aussi de la qualité du travail ou des dossiers auxquels on a accès. Comme les autres femmes, les avocates ont donc un prix à payer pour la maternité53, et même si certaines mettent en œuvre des stratégies de contournement (planification, report et suppression d'activités) comme nous l'avons vu plus haut, cela ne suffit pas toujours à vaincre les obstacles qui se dressent devant les femmes, ce qu'une auteure a appelé le « maternal wall » ${ }^{54}$.

51. Diane-Gabrielle Tremblay et Émilie Genin, « Parental leave: an important employee right, but an organizational challenge » (2011) 23:4 Employee Responsibilities and Rights Journal 249; DianeGabrielle Tremblay et Émilie Genin, « Parental Leave: When First Hand Experience Does Not Measure Up to Perception » (2010) 30:9/10 International Journal of Sociology and Social Policy 532.

52. Michelle J Budig et Paula England, « The Wage Penalty for Motherhood » (2001) 66:2 American Sociological Review 204.

53. Shoba V Arun, Thankom G Arun, et Vani K Borooah, « The Effect of Career Breaks on the Working Lives of Women » (2004) 10:1 Feminist Economics 65.

54. Nicole Buonocore Porter, «Re-Defining Superwoman: An Essay on Overcoming the "Maternal Wall" in the Legal Workplace » (2006) 13 Duke Journal of Gender Law and Policy 55. 
Copyright of Canadian Journal of Women \& the Law is the property of UTP/Canadian Journal of Women \& the Law and its content may not be copied or emailed to multiple sites or posted to a listserv without the copyright holder's express written permission. However, users may print, download, or email articles for individual use. 